ORIGINAL ARTICLE

\title{
A randomised controlled trial of morphine versus phenobarbitone for neonatal abstinence syndrome
}

\author{
L Jackson, A Ting, S Mckay, P Galea, C Skeoch
}

Arch Dis Child Fetal Neonatal Ed 2004;89:F300-F304. doi: 10.1136/adc.2003.033555

See end of article for authors' affiliations

....................

Correspondence to: Dr Jackson, Neonatal Unit, Princess Royal Maternity Hospital, Glasgow Royal Infirmary, 84 Castle Street, Glasgow G4 OSF,

Scotland, UK; LesleyJackson@ yahoo.co.uk

Accepted 13 August 2003

\begin{abstract}
Background: The incidence of neonatal abstinence syndrome (NAS) has increased 10-fold over the last decade in Glasgow. In the Princess Royal Maternity Hospital, it now accounts for $17 \%$ of special care baby unit (SCBU) admissions.

Objective: To compare opiate replacement therapy (morphine sulphate) with the present standard treatment (phenobarbitone) for management of NAS. The primary study end point was duration of pharmaceutical treatment. Secondary end points were the requirement for additional drugs and the requirement for SCBU admission.

Design: Double blind, randomised controlled clinical trial.

Methods: Differential diagnoses were excluded, and two consecutive Lipsitz scores $>4$ defined NAS requiring treatment. Infants were randomised to receive morphine sulphate or phenobarbitone. Treatments were identical in appearance, odour, and volume. Increments, decrements, and discontinuation of treatments were protocol driven.

Results: Seventy five infants participated. All mothers received opiate replacement therapy (methadone) during pregnancy and most used other drugs ( $n=62,83 \%$ ). No significant difference in maternal drug use patterns was observed between treatment groups. Median treatment duration was four days shorter with opiate replacement ( $8 v 12$ days, Mann-Whitney $U$ test, $p=0.02)$. Phenobarbitone treated infants tended to require second line treatment $\left(47 \% v 35 \%, \chi^{2}\right.$ test, $\left.p=0.11\right)$ and SCBU admission $(62 \% \vee 30 \%$, $\chi^{2}$ test, $\mathrm{p}=0.04$ ) more often.

Conclusions: Opiate replacement therapy appears to be superior for management of symptomatic NAS when maternal opiate use is prevalent. The shorter treatment duration and lower requirement for higher intensity nursing may have significant cost implications. Tailoring NAS treatment to local maternal drug use may result in similar benefits.
\end{abstract}

$\mathrm{N}$ eonatal abstinence syndrome (NAS) is a syndrome of drug withdrawal observed in infants delivered to mothers who are physically dependent on addictive drugs during their pregnancies, manifesting as non-specific symptoms and signs, of which irritability, poor feeding with an inadequate suck and high pitched crying are common. Rarely, seizures may occur and there is an increased neonatal mortality from sudden infant death syndrome in the short term, in addition to a long term adverse neurodevelopmental outcome. ${ }^{12}$

NAS is increasing in incidence world wide. In the United States, the number of drug affected newborns has increased by $300 \%$ since the 1980s. ${ }^{3}$ In the United Kingdom, the incidence of drug exposed newborn infants varies between $14 \%$ and $90 \%$ of liveborn infants, depending on the urban area and social class of the population sampled. ${ }^{4}$ More recently, anonymous screening of women attending antenatal clinics in London observed that $11-16 \%$ were using at least one illicit substance. ${ }^{56}$ In the area served by Glasgow Royal Maternity Hospital, NAS has increased 10-fold over the last decade, paralleling the increased usage of methadone as opiate substitution for pregnant mothers with a history of drug use. NAS at present accounts for $17 \%$ of admissions to the special care baby unit (SCBU). ${ }^{78}$ In addition, local audit suggests that symptomatic NAS has become increasingly difficult to treat, requiring multiple pharmaceutical treatments administered for longer durations. The increased prevalence of NAS has clear implications for mother-infant bonding, cot occupancy, nursing time, and costs.

Despite the increasing clinical burden of NAS, optimal management remains unclear as clinical trial evidence is insufficient. Conservative measures such as holding and minimal stimulation may suffice if symptoms are mild and non-progressive. However, more severe symptoms require adequate pharmacotherapy. Many pharmaceutical agents have been used historically to treat NAS, including clonidine, chloral hydrate, chlorpromazine, opioids, opiates, and phenobarbitone. ${ }^{10}$ Studies to date can be criticised on their lack of standardisation of outcome measures, problems with randomisation, and failure to use a pre-evaluated scoring system to allow standardisation of the start of treatment, dosage alterations, and termination of pharmaceutical treatment. ${ }^{11-13}$

The aim of this study was to compare the efficacy of initial treatment with an opiate versus phenobarbitone for infants with NAS caused by opiate withdrawal. The total duration of pharmaceutical treatment required to achieve symptom resolution was predefined as the primary study end point, and the requirement for higher intensity nursing in the SCBU or the need for an additional second line treatment as secondary end points.

\section{SUBJECTS AND METHODS}

Phenobarbitone has been the standard first line treatment for symptomatic NAS at Glasgow Royal Maternity Hospital for many years. Oral morphine sulphate was selected as a treatment option for this study as opiates are the predominant class of drugs used by drug dependent pregnant women in Glasgow. Statistical advice was sought, and a sample size of 80 was estimated to detect a 0.5 SD difference in the total

Abbreviations: NAS, neonatal abstinence syndrome; SCBU, special care baby unit 
duration of pharmacological treatment between the treatment groups, assuming $\alpha=0.05$. After informed written parental consent had been obtained, infants with a history of maternal drug use and two sequential scores of $>4$ using the Lipsitz tool ${ }^{14}$ were considered eligible for randomisation. Alternative diagnoses for an irritable baby were excluded by clinical examination and biochemical analysis (including calcium, magnesium, and blood glucose concentrations). The Lipsitz tool was selected as the measure of NAS severity as it is the scoring system recommended by the American Academy of Pediatrics, ${ }^{3}$ is less labour and time intensive than alternative scoring systems in use, ${ }^{15-18}$ and has a sensitivity approaching $80 \%$ for NAS requiring pharmaceutical treatment when a score of $>4$ is used..$^{14}$ Six months before the start of the study, all midwifery staff in both the postnatal ward and SCBU were trained in its use and were well accustomed to treatment alterations based on this score by the time the study started. Case notes were also retrospectively reviewed to record Apgar scores at one and five minutes, in case infant irritability reflected acute compromise at the time of delivery. Infants were recruited on the postnatal ward or after admission to SCBU if this was required for any other reason such as prematurity. Local clinical guidelines dictated that infants with NAS were treated in the postnatal ward with their mothers unless they required nasogastric feeds, had severe withdrawal, were admitted to SCBU for another reason such as prematurity/ sepsis, or their mothers had already been discharged on the 10th postnatal day. Seventy six infants with symptomatic NAS fulfilling the above criteria were eligible for recruitment for study between February 2000 and January 2001. Parental permission was refused for one infant, so 75 infants were recruited. Table 1 summarises the clinical characteristics of those studied.

Infants were randomised to receive morphine sulphate or phenobarbitone orally, labelled as substance A and substance B respectively. Randomisation was performed using a computer generated random number technique. Morphine sulphate was prescribed in a dose of $50 \mu \mathrm{g} / \mathrm{kg}$ and phenobarbitone in a dose of $2 \mathrm{mg} / \mathrm{kg}$. Both drugs were administered four times a day as identical colourless and odourless solutions of equal volume. Solutions A and B were specifically prepared for this study by Tayside Pharmaceuticals, Ninewells Hospital, Dundee, Scotland, UK. Throughout the study period, all staff involved remained unaware of the identity of either treatment. To ensure objectivity, the start, dosage increments and decrements, and eventual termination of treatment were protocol driven (fig 1) based on sequential 12 hourly postprandial Lipsitz scores, which were obtained throughout the period of study. The start and

Table 1 Clinical characteristics of the infants studied and classes of drugs used by their mothers

\begin{tabular}{lll}
\hline & $\begin{array}{l}\text { Oramorph } \\
(\mathbf{n}=\mathbf{4 1})\end{array}$ & $\begin{array}{l}\text { Phenobarbitone } \\
(\mathbf{n}=34)\end{array}$ \\
\hline Gestational age (weeks) & $40(32-42)$ & $39(33-41)$ \\
Birth weight (g) & $2960(1760-$ & $2780(1860-$ \\
& $3930)$ & $3760)$ \\
Age randomised (hours) & $48(12-120)$ & $48(12-240)$ \\
Breast fed (\%) & 4.9 & 5.9 \\
Methadone dose (mg) & $30(5-65)$ & $35(10-100)$ \\
$\quad$ Interquartile range & $15-49$ & $24-51$ \\
Opiate exposed (\%) & 100 & 100 \\
Benzodiazepine exposed (\%) & 22 & 44 \\
Exposed to other drug classes (\%) & 10 & 23 \\
Apgar 1 minute & $9(5-10)$ & $9(4-9)$ \\
Apgar 5 minutes & $10(8-10)$ & $9(6-10)$ \\
\hline Where applicable, values are mean (range). &
\end{tabular}

discontinuation of second line treatment was similarly protocol driven.

Statistical analysis was performed using SPSS for Windows (SPSS Inc, Chicago, Illinois, USA). The relation between demographic factors and the primary outcome variable (total duration of pharmaceutical treatment) was examined initially using non-parametric tests. Demographic factors correlating with the primary outcome were entered into a univariate linear model (general linear modelling, analysis of covariance) to account for potential confounding influences. This technique was used to determine the independent influence of the treatment allocation (A or B) on the duration of pharmaceutical treatment. The model used included treatment allocation and use of other non-opiate based drugs as between subjects factors, and the maternal methadone dose as a covariate, as these correlated significantly with the primary outcome. For the secondary outcome analyses (requirement for second line treatment, admission to SCBU required), non-parametric tests were used to determine which factors and covariates influenced these outcomes. Logistic regression was used to identify which of these factors independently influenced the secondary outcomes. Throughout the analysis, two tailed tests were used, non-normally distributed data were log transformed to normality, and significance was assumed at $\alpha=0.05$.

\section{RESULTS}

\section{Drugs misused by mothers}

All mothers of the infants in this study were using methadone as part of a harm reduction policy. Table 1 summarises the classes of drugs that infants were exposed to in utero. No significant differences were observed between those randomised to either treatment group (morphine sulphate/ phenobarbitone) in the dose of methadone used by mothers during their pregnancy. Most mothers used other drugs in addition to the methadone substitution provided during pregnancy $(\mathrm{n}=62,82.7 \%)$. The other classes of drugs used by mothers were predominantly benzodiazepines and a variety of others, which included antidepressants, neuroleptics, and cannabis. Cocaine use was uncommon. Table 1 summarises the proportions of infants exposed in utero to these other classes of drugs. Infants randomly allocated to receive phenobarbitone tended to have been exposed to benzodiazepines and other classes of drugs more often than those randomly allocated to receive morphine sulphate.

\section{Effect of treatment allocation on study outcomes}

The primary outcome measure was the duration of pharmaceutical treatment required to objectively resolve the symptoms of NAS. Infants randomised to receive morphine sulphate required a median of four fewer days active treatment than those allocated to phenobarbitone ( $8 v 12$ days, MannWhitney, $\mathrm{p}=0.02$ ). Maternal methadone dose (Spearman's $\rho=0.24, p=0.04)$ and classes of drugs used other than opiates $(\rho=0.24, p=0.41)$ also correlated with total days receiving treatment. When these significant influences were accounted for using linear modelling (analysis of covariance), treatment allocation remained a significant independent predictor of the total duration of pharmaceutical treatment $(\mathrm{p}=0.03)$. Maternal methadone dose also independently influenced the duration of treatment $(p=0.04)$, although the use of other classes of drugs did not.

Secondary outcome measures were the requirement for an additional second line treatment to adequately suppress symptoms of NAS (chloral hydrate $15 \mathrm{mg} / \mathrm{kg}$ ), and the requirement for admission to the SCBU. Infants receiving phenobarbitone as their primary treatment tended to require an additional drug more often to suppress NAS symptoms $\left(47 \% \vee 35 \%, \chi^{2}\right.$ test $\left.\mathrm{p}=0.11\right)$. Other factors also appeared to 


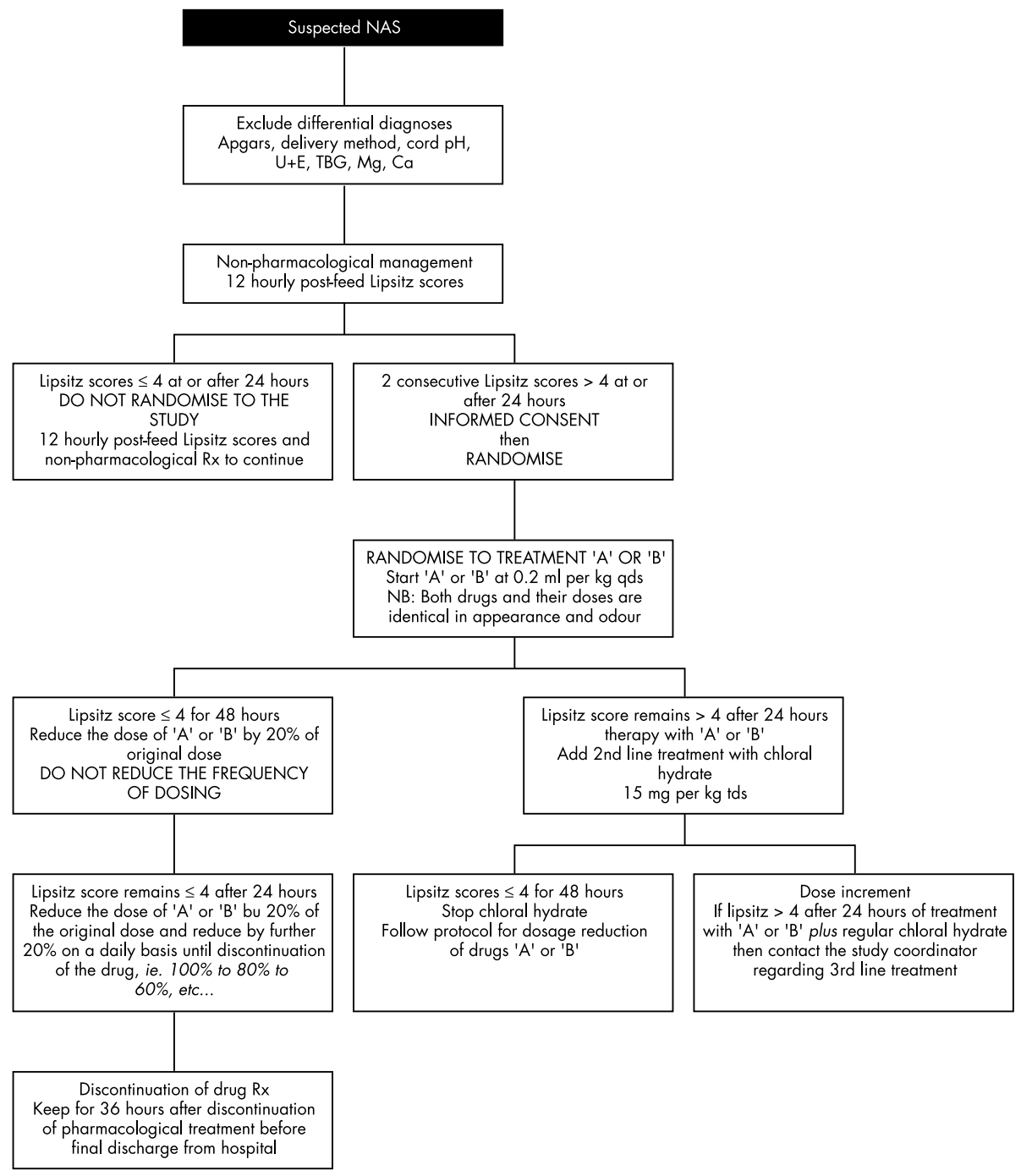

Figure 1 Flow chart summarising the study protocol for start, discontinuation, and dosage adjustment of treatments. NAS, Neonatal abstinence syndrome; TBG, true blood glucose; Rx, prescription; $U+E$, urea and electrolytes.

correlate with the requirement for second line treatment. These included the reported maternal methadone dose $(\rho=0.32, p<0.01)$, in utero exposure to classes of drugs other than opiates or benzodiazepines $(\rho=0.27, \mathrm{p}=0.02)$, and exposure to benzodiazepines $(\rho=0.24, p=0.04)$. To determine the independent effect of treatment allocation on the requirement for second line therapy, logistic regression was used. Treatment allocation did not independently predict the requirement for second line therapy $(p=0.34)$, which was only significantly predicted by maternal methadone dose $(\mathrm{p}=0.02)$.

The requirement for admission to SCBU, a subjective surrogate marker of more severe drug withdrawal, appeared to be lower in infants who received morphine sulphate as their primary treatment $\left(30 \% v 62 \%, \chi^{2}\right.$ test $\left.\mathrm{p}=0.04\right)$. However, other factors also appeared to correlate with the requirement for SCBU admission. These included the reported maternal methadone dose $(\rho=0.27, p=0.02)$, exposure to classes of drug other than opiates or benzodiazepines in utero $(\rho=0.27, p=0.02)$, and the treatment allocated $(\rho=0.27$, $\mathrm{p}=0.02$ ). The treatment allocated (morphine sulphate/ phenobarbitone) independently predicted the requirement for SCBU admission ( $p=0.04)$ as did the maternal methadone dose $(p=0.04)$. However, the duration of SCBU stay was not predicted by any of the above factors.

\section{DISCUSSION}

In this study, tailoring the treatment for symptomatic NAS to match the local pattern of maternal drug use was associated with two important benefits: (a) a more rapid resolution of the symptoms and signs of NAS, such that treatment was required for a shorter time; $(b)$ a significantly reduced requirement for higher intensity nursing, implying more effective treatment. These two benefits are likely to translate into reductions in cot occupancy (estimated at about $300 \mathrm{cot}$ days per annum, based on 80 cases of NAS per annum in our centre) and midwifery staff time. Both will have considerable cost implications. However, to replicate these findings it is important to understand local maternal drug use.

Data comparing the use of opiates with other agents to treat symptomatic NAS are limited. Pacifico et al ${ }^{10}$ concluded that morphine alone was superior to the combination of phenobarbitone and diazepam, and to the combination of morphine, phenobarbitone, and diazepam. Unfortunately, the report contains insufficient detail on its study design to be easily compared with our study. Madden et al ${ }^{19}$ found no significant difference between treatments consisting of methadone, phenobarbitone, or diazepam, although treatment decisions were made subjectively without the aid of a standardised chart, which introduces the possibility of bias. Few studies are directly comparable with our findings. 
In this study, several weaknesses must be noted. No loading dose of phenobarbitone was used to ensure that the drug regimens were blinded. This could potentially have introduced bias in favour of morphine sulphate. However, the necessity of phenobarbitone loading is debatable. Kaltenbach et $a l^{20}$ compared 36 drug exposed infants treated with phenobarbitone regimens that did or did not use a loading dose. No significant difference was identified between regimens. However, Finnegan et $a l^{21}$ observed a significantly reduced time to symptom control in infants receiving a loading dose of phenobarbitone. We acknowledge that the lack of a loading dose of phenobarbitone in our study may have contributed to the apparently poorer treatment outcomes in the phenobarbitone group.

In addition, our protocol did not allow for adjustments in either the frequency or dose of the allocated treatment. Kandall ${ }^{22}$ compared phenobarbitone with three hourly treatment with paregoric (an opiate based drug) and observed no difference in the treatment duration required. Khoo ${ }^{23}$ enrolled infants to treatment with morphine every four to six hours or phenobarbitone (loading dose and daily maintenance thereafter), but the methodology does not allow comparison with our randomised trial. The optimal frequency of opiate dosing for symptomatic NAS remains unclear. However, the dose of opiate administered in this study may be of more importance. Maternal methadone dose was an independent predictor of the duration of NAS treatment required, the requirement for second line treatment, and the requirement for SCBU admission (a subjective surrogate of severity of the withdrawal process). These observations suggest that the morphine sulphate dose used may have been insufficient, and therefore the benefits of opiate replacement therapy for symptomatic NAS may have been underestimated. Doberczak et $a^{24}$ observed that the maternal methadone dose at delivery correlated significantly with the neonatal plasma methadone concentration on day 1 of life, the severity of central nervous system signs of withdrawal, and the rate of decline of the neonatal plasma methadone concentration from day 1 to day 4 of life. Further work is planned locally to investigate whether a higher dose of opiate replacement for symptomatic NAS, particularly for infants whose mothers were receiving large methadone maintenance doses, has additional clinical benefit above that observed in this study.

In interpreting the findings, it is important to note that differences were reported in the pattern of use of other drugs (benzodiazepines and other classes) between those randomly allocated to receive either morphine sulphate or phenobarbitone, with the phenobarbitone group exposed significantly more often to benzodiazepines (44\% v22\%) and tending to have been exposed more often to other classes of drugs $(23 \%$ $v 10 \%)$. However, when statistical methods that adjusted for these differences were used (linear modelling, logistic regression), in utero exposure to benzodiazepines and other classes of drugs did not appear to independently influence any of the predefined study outcomes. This implies that the differences observed in the maternal use of these other drugs were not of great clinical importance to the outcome of the present study. We recorded the maternal drug history, but did not perform further analytical techniques to validate it. Modern analytical techniques such as meconium analysis ${ }^{25}{ }^{26}$ may have allowed a more definitive estimation of in utero drug exposure.

The aims of this study were confined to inpatient outcomes within the neonatal period. Exploration of the long term neurodevelopmental effects of the study protocol (randomisation of infants to receive either phenobarbitone or morphine sulphate) was not its purpose, and the long term morbidity from neonatal drug withdrawal remains relatively unstudied. Few studies have followed drug exposed children beyond the first few years of life, as confounding variables, such as environment and dysfunctional caregivers, make it extremely difficult to determine the causes of differences in ability. It is hoped to address this lack of long term data by re-examining at primary school age the present cohort and an environmentally matched control group not exposed to drugs, with a battery of cognitive ability tests as the primary outcome.

In conclusion, this study suggests that opiate replacement therapy for symptomatic NAS can achieve meaningful clinical benefits in a population in which maternal use of drugs from the opiate class is predominant. This policy more effectively suppressed the underlying disease process of NAS, thereby reducing the time to achieve adequate symptom control. Further studies are required to determine the optimal regimen and dose, and meconium analysis ${ }^{25}{ }^{26}$ may be useful for tailoring the clinical management of these infants and may give further information on whether in utero polydrug exposure is of clinical importance. The potential benefit of long acting opiates (such as methadone) that can be administered from time to time requires exploration, as an opportunity for domiciliary pharmaceutical treatment may exist. These thoughts for the future will require formal clinical assessment in a randomised clinical trial.

\section{ACKNOWLEDGEMENTS}

We acknowledge the Greater Glasgow Health Board for funding and all medical and nursing staff who participated in the study.

\section{Authors' affiliations}

L Jackson, A Ting, S Mckay, P Galea, C Skeoch, Neonatal Unit, Princess Royal Maternity Hospital, Glasgow Royal Infirmary, Glasgow, Scotland, UK

\section{REFERENCES}

1 Ornoy A, Michailevskaya V, Lukashov I, et al. The developmental outcome of children born to heroin-dependent mothers, raised at home or adopted. Child Abuse Negl 1996;20:385-96.

2 Ornoy A, Segal J, Bar-Hamburger R, et al. Developmental outcome of schoolage children born to mothers with heroin dependency: importance of environmental factors. Dev Med Child Neurol 2001;43:668-75.

3 American Academy of Pediatrics Committee on Drugs. Neonatal drug withdrawal. Pediatrics 1998;101:1079-88.

4 Morrison CL, Siney C. A survey of the management of neonatal opiate withdrawal in England and Wales. Eur J Pediatr 1996;155:323-6.

5 Sherwood RA, Keating J, Kavvadia V, et al. Substance misuse in early pregnancy and relationship to fetal outcome. Eur J Pediatr 1999;158:488-92.

6 Farkas AG, Colbert DL, Erskine KJ. Anonymous testing for drug abuse in an antenatal population. Br J Obstet Gynaecol 1995;102:563-5.

7 Shaw NJ, Mclvor L. Neonatal abstinence syndrome after maternal methadone treatment. Arch Dis Child Fetal Neonatal Ed 1994;71:F203-5.

8 Alroomi LG, Davidson J, Evans TJ, et al. Maternal narcotic abuse and the newborn. Arch Dis Child 1988;63:81-3.

9 Hoder EL, Leckman JF, Poulsen J, et al. Clonidine treatment of neonatal narcotic abstinence syndrome. Psychiatry Res 1984;13:243-51.

10 Pacifico P, Nardelli E, Pantarotto MF. Neonatal heroin withdrawal syndrome: evaluation of different pharmacological treatments. Pharmacol Res 1989;21(suppl 1):63-4.

11 Theis JG, Selby P, Ikizler Y, et al. Current management of the neonatal abstinence syndrome: a critical analysis of the evidence. Biol Neonate 1997;71:345-56

12 Osborn DA, Cole MJ, Jeffery HE. Opiate treatment for opiate withdrawal in newborn infants. Cochrane Database Syst Rev 2002:CD002059.

13 Osborn DA, Jeffery HE, Cole MJ. Sedatives for opiate withdrawal in newborn infants. Cochrane Database Syst Rev 2002:CD002053.

14 Lipsitz PJ. A proposed narcotic withdrawal score for use with newborn infants. A pragmatic evaluation of its efficacy. Clin Pediatr (Phila) 1975; 14:592-4.

15 Finnegan LP, Connaughton JF Jr, Kron RE, et al. Neonatal abstinence syndrome: assessment and management. Addict Dis 1975;2:141-58.

16 Rivers RP. Neonatal opiate withdrawal. Arch Dis Child 1986;61: 1236-9.

17 Zahorodny W, Rom C, Whitney W, et al. The neonatal withdrawal inventory: a simplified score of newborn withdrawal. J Dev Behav Pediatr 1998; 19:89-93.

18 Green M, Suffet F. The Neonatal Narcotic Withdrawal Index: a device for the improvement of care in the abstinence syndrome. Am J Drug Alcohol Abuse $1981 ; 8: 203-13$. 
19 Madden JD, Chappel JN, Zuspan F, et al. Observation and treatment of neonatal narcotic withdrawal. Am J Obstet Gynecol 1977; 127:199-201

20 Kaltenbach K, Finnegan LP. Neonatal abstinence syndrome, pharmacotherapy and developmental outcome. Neurobehav Toxicol Teratol 1986;8:353-5.

21 Finnegan LP, Mitros TF, Hopkins LE. Management of neonatal narcotic abstinence utilizing a phenobarbital loading dose method. NIDA Res Monogr 1979;27:247-53

22 Kandall SR. Treatment strategies for drug-exposed neonates. Clin Perinatol 1999;26:231-43.
23 Khoo BH. Neonatal narcotic drug withdrawal syndrome. Med J Malaysia 1978;32:297-301

24 Doberczak TM, Kandall SR, Friedmann P. Relationship between maternal methadone dosage, maternal-neonatal methadone levels, and neonatal withdrawal. Obstet Gynecol 1993;81:936-40.

25 Ostrea EM, Knapp DK, Knapp DK, et al. Estimates of illicit drug use during pregnancy by maternal interview, hair analysis, and meconium analysis. J Pediatr 2001;138:344-8

26 Ostrea EM, Brady M, Cause S, et al. Drug screening of newborns by meconium analysis: a large-scale, prospective, epidemiologic study. Pediatrics 1992;89:107-13. 